\title{
New approach of solving time-domain free space wave propagation
}

\begin{abstract}
In this paper, a numerical simulation by a new high speed low order FDTD (HSLO-FDTD) method will be conducted to simulate one dimensional free space wave propagation of 2.4 $\mathrm{GHz}$ Gaussian pulse. The efficiency of the new schemes are analyze and compared with the standard FDTD method in terms of processing time, phase velocity and global error. The amplitude in volts by both methods is also displayed. Results obtained using the new schemes compare well with published results and solve the problem faster than the standard FDTD method.
\end{abstract}

Keyword: FDTD; HSLO-FDTD; Numerical simulation of wave propagation 